\title{
Meta-Analysis
}

\section{e High Frequency Repetitive Transcranial Magnetic Stimulation Therapy For Chronic Neuropathic Pain: A Meta-analysis}

\footnotetext{
From: ${ }^{1}$ Luzhou Medical College, China; ${ }^{2}$ Imaging Institute of Rehabilitation and Development of Brain Function, the Second Clinical Medical College of North Sichuan Medical College Nanchong Central Hospital, China; ${ }^{3}$ Lotus Biotech.com LLC, John Hopkins University-MCC, Rockville, MD; ${ }^{4}$ Peking University Third Hospital, Beijing, China

Address Correspondence: Qiwen Mu, MD, PhD Imaging Institute of Rehabilitation and Development of Brain Function

North Sichuan Medical University Nanchong Central Hospital

97 South Renmin Road, Shunqing District, Nanchong 637000 Sichuan, China; Peking University Third Hospital, 49 North Garden Road Haidian District Beijing 100191, China E-mail: muqiwen99@yahoo.com

Disclaimer: There was no external funding in the preparation of this manuscript. Conflict of interest: Each author certifies that he or she, or a member of his or her immediate family, has no commercial association (i.e., consultancies, stock ownership, equity interest, patent/licensing arrangements, etc.) that might pose a conflict of interest in connection with the submitted manuscript.

Manuscript received: 04-14-2015 Revised manuscript received: 06-09-2015

Accepted for publication: 06-22-2015

Free full manuscript: www.painphysicianjournal.com
}

Yu Jin, MS ${ }^{1,2}$, Guoqiang Xing, $\mathrm{PhD}^{3}$, Guangming $\mathrm{Li}, \mathrm{PhD}^{2}$, Anguo Wang, $\mathrm{MS}^{2}$,

Shenggang Feng, $\mathrm{PhD}^{2}$, Qing Tang, $\mathrm{MS}^{2}$, Xiang Liao, $\mathrm{MS}^{2}$, Zhiwei Guo, $\mathrm{MS}^{2}$, Morgan A. McClure, MS², and Qiwen Mu, MD, PhD ${ }^{1,2,4}$

Background: Increasing evidence supports an analgesic effect of repetitive transcranial magnetic stimulation (rTMS) for neuropathic pain (NP). However, the optimal parameters of rTMS (stimulation frequency and treatment sessions) for achieving long-term analgesic effects remain unknown. This study analyzed the current findings in the literature.

Objective: The aim of this study was to assess the optimal parameters of rTMS for NP, including the rTMS sessions needed for inducing acute as well as long-term analgesic effects.

Study Design: A meta-analysis of the analgesic effect of high frequency rTMS (HF- rTMS) for neuropathic patients.

Setting: This meta-analysis examined all studies involving the analgesic efficacy of HF-rTMS for NP.

Methods: PubMed, Embase, and the Cochrane library were searched for clinical studies of rTMS treatment on NP published before December 31, 2014. Crude standardized mean differences (SMD) with $95 \%$ confidence interval $(\mathrm{Cl})$ were calculated for pain intensity after different treatment sessions (from 1 to 10) and follow-up of one or 2 months after rTMS treatment using random effect models.

Results: Twenty-five studies (including 32 trials and 589 patients) were selected for the metaanalysis according to the inclusion and exclusion criteria. All 3 HF-rTMS treatments (5, 10, and 20 $\mathrm{Hz}$ ) produced pain reduction, while there were no differences between them, with the maximal pain reduction found after one and 5 sessions of rTMS treatment. Further, this significant analgesic effect remained forone month after 5 sessions of rTMS treatment.

Limitations: There are limitations of this meta-analysis. For example, the long-term analgesic effects of different HF-rTMS and low frequency (LF) rTMS sessions, including the single session of rTMS on different NP of varying origins have yet not been evaluated; the full degree of pain relief is still unclear for many rTMS studies.

Conclusions: HF-rTMS stimulation on primary motor cortex is effective in relieving pain in NP patients. Although 5 sessions of rTMS treatment produced a maximal analgesic effect and may be maintained for at least one month, further large-scale and well-controlled trials are needed to determine if this enhanced effect is specific to certain types of NP such as post-stroke related central NP.

Key words: High frequency, repetitive transcranial magnetic stimulation, neuropathic pain, single stimulation, multiple stimulation, meta-analysis

Pain Physician 2015; 18:E1029-E1046 
$\mathbf{N}$ europathic pain (NP) is a localized sensation of unpleasant discomfort caused by damage or disease in the peripheral (peripheral neuropathic pain) and/or central (central neuropathic pain) nervous system that affects the somatosensory system (1). NP may include abnormal sensations (dysesthesia), and pain from normally non-painful stimuli (allodynia). It may resemble stabbings or electric shocks, burning or coldness, "pins and needles" sensations, numbness, and itching. The International Association for the Study of Pain (IASP) defined NP as follows: "pain arising as a direct consequence of a lesion or disease affecting the somatosensory system" (2). The annual incidence of NP has been reported to be $0.82 \%$ (3), and the prevalence of chronic NP has been reported as between 7 and $8 \%$ in the general population $(4,5)$. Despite the IASP's recommendation of a 3-line drug treatment for NP (6), these drugs are often ineffective and high doses are required for achieving partial pain relief. In a recent meta-analysis of evidence-based pain studies, Finnerup et al (7) reported that only 30 - $50 \%$ of the patients responded to pharmacological treatment and only with $30-40 \%$ pain reduction (8).

The major characteristic of NP is neuronal overexcitability resulting from a combination of multiple factors that contribute to the diverse features of NP under various medical conditions (6). NP could arise from peripheral sensitization or abnormal central sensitization. After peripheral nerve lesion, aberrant regeneration may lead to abnormal neuronal excitability, and heightened sensitivity to various stimuli (9). The spinal cord dorsal horn neurons of the spinothalamic tract (STT) constitute the major ascending nociceptive pathway. As a consequence of ongoing spontaneous activity arising in the periphery, STT neurons develop increased background activity, enlarged receptive fields, and increased responses to afferent impulses, including normally innocuous tactile stimuli. Changes in impulse processing in the dorsal horns of the spinal cord, due to the release of neurotransmitters after the nerve damage or loss of the balance between the activities of the ascending excitatory systems and the descending inhibitory systems caused by various diseases in central nervous system has been supposed as the pathological mechanism of NP (10).

Because primary motor cortex stimulation (MCS) with surgically implanted epidural electrodes has been found to produce analgesic effects in about half of NP patients (11), and because a non-invasive coil-generated pulsating magnetic field near the cortex could induce electric currents to modulate nerve cell activity in focal brain areas (12), it was proposed that repetitive transcranial magnetic stimulation (rTMS) could be a safe method to modulate cortical excitability and pain threshold $(13,14)$.

As summarized in recent reviews, high frequency rTMS (HF-rTMS) applied over the primary motor cortex (M1) could alleviate NP of various origins, such as spinal cord injury ( $\mathrm{SCl})$, post-stroke, amputation, and brachial avulsion pain (15-17). In a randomized, double-blind, sham-controlled crossover multi-center study, Hosomi et al (18) showed that HF-rTMS $(5 \mathrm{~Hz})$ on M1 caused modest pain reduction in people with NP. However, the optimal number of rTMS sessions and the duration of rTMS-induced analgesic effect have not been reported.

Previous meta-analyses of NP mainly focused on the neuroanatomical origins or the chronic nature of NP $(16,17)$. The aim of this study was to assess the optimal parameters of rTMS for NP, including the rTMS sessions needed for inducing acute as well as long-term analgesic effects. Only HF-rTMS $(>1 \mathrm{~Hz})$ studies were included due to the data availability.

\section{Methods}

\section{Literature Search}

A systematic search of the literature was performed to identify the relevant clinical studies. PubMed, Embase, and the Cochrane Library were searched up to 31 December, 2014. Search terms were selected to link NP and rTMS. Keywords were ("repetitive transcranial magnetic stimulation" or "rTMS" or "TMS") and ("pain" or "neuropathic pain").

\section{Study Selection}

The selected studies, based on their title and abstract, had to meet the following criteria:

1. Types of studies: randomized sham-controlled or self-controlled trials; double-blind or single-blind; parallel or cross-over study designs;

2. $\geq 5$ patients in a trial;

3. Characteristics of NP patients: all patients must be 18 years of age or older, and clearly diagnosed according to the criteria for NP by the IASP $(6,19)$. If an etiological factor is known, it should be included, e.g., diabetic neuropathic pain, post-herpetic neuropathic pain, central post-stroke pain, or neuropathic pain following $\mathrm{SCI}$. In addition, other types of pain should be taken into account as long as NP was reported as the most disturbing pain (20); 
4. Types of intervention: HF-rTMS ( $>1 \mathrm{~Hz})$ over the M1 by single or multiple rTMS stimulation.

5. Publication limit: articles were human studies and written in English;

6. Types of outcome: Pain level was self-scored by the patient assessed on a $0-10$ visual analog scale (VAS) or $0-10$ numerical rating scale (NRS) after each session. The VAS consists of a $15 \mathrm{~cm}$ plastic ruler with a slider in the middle, with values from 0 to 10 . The 2 sides represented "no pain sensation" ( 0 on the VAS scale) and "the most intense pain" (10 on the VAS scale) (21). The NRS was used digitally from 0 to 10 to represent pain intensity. The endpoints were set as "no pain sensation" ( 0 on the NRS) and "the most intense pain sensation imaginable" (10 on the NRS) (22). The analgesic effects of rTMS were estimated by comparing the pain scores obtained before and after the intervention, or by comparing the experimental group with the sham group using VAS or NRS scores, whenever applicable;

7. The article must contain the original data (VAS or NRS score), or data could be extracted and calculated based on the existing materials.

\section{Exclusion criteria:}

1. Articles did not meet the above criteria;

2. Studies did not provide pre-treatment score, and/or the score of sham group for NP;

3. The outcomes were not described as a mean \pm standard deviation (SD) or mean \pm standard errors of the mean (SEM), but as median or inter-quartile range.

\section{Trial Quality Appraisal}

Two reviewers independently assessed the methodological qualities of the included studies based on a modified checklist from Moher et al (23) that offered the evaluation criteria as shown in Table 1. If there was a disagreement, a third researcher re-assessed the article and discussed it with the 2 reviewers to reach an agreement. If the patients were randomly allocated, it was recorded as 1 . In scoring blinding, 0 represented a non-blind or no-mention in the article, and 1 and 2 represented single-blind and double-blind, respectively. Dropouts were recorded as the numbers of patients who had withdrawn before the end of the study. Descriptions of baseline demographic data were recorded as 1 if described. The numbers and types of adverse effects were recorded.
Table 1. Quality of the included studies.

\begin{tabular}{|c|c|c|c|c|c|c|c|}
\hline & Study & (1) & (2) & (3) & (4) & (5) & (6) \\
\hline 1 & Ahmed 2011 & 1 & 0 & 0 & 1 & 1 & 0 \\
\hline 2 & André-Obadia 2006 & 1 & 2 & 2 & 1 & 1 & 0 \\
\hline 3 & André-Obadia 2008 & 1 & 2 & 2 & 1 & 1 & 0 \\
\hline 4 & André-Obadia 2011 & 1 & 1 & 0 & 1 & 1 & 0 \\
\hline 5 & Defrin 2007 & 1 & 2 & 0 & 1 & 1 & 0 \\
\hline 6 & Goto 2008 & 0 & 0 & 0 & 1 & 1 & 0 \\
\hline 7 & Hirayama 2006 & 1 & 1 & 0 & 1 & 1 & 0 \\
\hline 8 & Hosomi $2013^{1}$ & 0 & 0 & 0 & 1 & 1 & 0 \\
\hline 9 & Hosomi $2013^{2}$ & 1 & 2 & 3 & 1 & 1 & 12 \\
\hline 10 & Jetté 2013 & 1 & 2 & 2 & 1 & 1 & 0 \\
\hline 11 & Kang 2009 & 1 & 2 & 0 & 1 & 1 & 0 \\
\hline 12 & Khedr 2005 & 1 & 0 & 0 & 1 & 1 & 0 \\
\hline 13 & Khedr 2014 & 1 & 1 & 0 & 1 & 1 & 0 \\
\hline 14 & Lefaucheur 20011 & 1 & 1 & 0 & 1 & 1 & 0 \\
\hline 15 & Lefaucheur 20012 & 1 & 2 & 0 & 1 & 1 & 0 \\
\hline 16 & Lefaucheur 2006 & 1 & 1 & 0 & 1 & 1 & 0 \\
\hline 17 & Lefaucheur 2008 & 1 & 2 & 0 & 1 & 1 & 0 \\
\hline 18 & Lefaucheur 2012 & 0 & 0 & 0 & 1 & 1 & 0 \\
\hline 19 & Matsumura 2013 & 1 & 0 & 0 & 1 & 1 & 0 \\
\hline 20 & Ohn 2012 & 0 & 0 & 0 & 1 & 1 & 0 \\
\hline 21 & Onesti 2013 & 1 & 2 & 2 & 1 & 1 & 0 \\
\hline 22 & Picarelli 2010 & 1 & 2 & 1 & 1 & 1 & 23 \\
\hline 23 & Pleger 2004 & 0 & 0 & 0 & 1 & 1 & 0 \\
\hline 24 & Rollink 2002 & 1 & 0 & 0 & 1 & 1 & 0 \\
\hline 25 & Saitoh 2007 & 1 & 0 & 0 & 1 & 1 & 0 \\
\hline
\end{tabular}

(1) random allocation, (2) blinding procedure, (3) dropout number, (4) description of baseline demographic data, (5) control study, (6) description of adverse events. 


\section{Data Extraction}

A standard form was jointly designed by both evaluators to collect basic information, which contains the following:

1. Patient characteristics: age (mean \pm SD), gender, number of participants, primary diagnosis (resulting in NP), and pain type;

2. Study design;

3. The parameters of rTMS: stimulation site, frequency, intensity (including the total pulses), and stimulation sessions (days);

4. Outcome measurement:

1. Mean and SD of post-stimulation pain score for both the rTMS treatment group and sham group.

2. If the study did not have a sham group, the pre-intervention data were used.

3. If the VAS was on a $0-100$ scale, it was also allowable.

4. If the outcome was expressed only as a graph, the software GetData Graph Digitizer 2.25 (http://getdata-graph-digitizer.com/) was used to extract the required data.

5. If the pain reduction was described as the rate relative to pre-intervention baseline of $100 \%$ or as the reduced rate, the following equations were used to calculate the post-intervention VAS score:

a). VAS\% = Post-treatment Pain Score / Pretreatment Pain Score $\times 100 \%$;

b). VAS reduction rate $(\%)=$ (Post-treatment Pain Score - Pre-treatment Pain Score) / Pretreatment Pain Score $\times 100 \%$.

\section{Data Synthesis and Analysis}

To evaluate the optimal number of rTMS sessions required for achieving significant pain reduction, the pain scores were extracted at the end of each of the following rTMS treatments: from the first session (one day session or one session) to the tenth session (10 day sessions). To evaluate long-term analgesic effects of rTMS, the follow-up observation was conducted at one and 2 months post rTMS treatment.

Meta-analysis was performed by using a Review Manager Software version 5.2 (Cochrane Collaboration, Oxford, England). The total analgesic effect-size of rTMS treatment, expressed by the standardized mean difference (SMD) with a 95\% confidence interval $(95 \%$ $\mathrm{Cl})$, was computed. The heterogeneity was tested using Q-statistics and the 12 index (24). If the 12 index was greater than $50 \%$, the random effect model was used for the analysis. Otherwise a fixed model was used. Finally, a funnel plot was constructed to test potential publication bias. A $P$ value deemed statistically significant was set at less than 0.05 .

\section{Results}

\section{Literature Search}

Of the total 470 studies found after the initial search, 25 studies were identified $(N=589)$ by 2 independent reviewers according to the inclusion and exclusion standards $(18,25-48)$. The flow diagram of the selection process is shown in Fig. 1.

Of the 25 studies selected for this meta-analysis, 5 studies were self-controlled $(30,32,40,42,45), 6$ studies were parallel sham controlled $(25,29,35,36,38,44)$, and 14 studies were crossover sham controlled $(18,26-$ $28,31,33,34,37,39,41,43,46-48)$, respectively. The duration time of NP ranged from 3 months to 122.4 months. The frequencies of rTMS applied were $5 \mathrm{~Hz}, 10 \mathrm{~Hz}$, and $20 \mathrm{~Hz}$. The stimulation site was the $\mathrm{M} 1$ corresponding to a painful region or contralateral to the painful site. For 18 studies, the pain scores of the experimental group were directly compared with those of the sham control group (18,25-29,33-39,43,44,46-48). The other studies compared the pain scores obtained before and after rTMS intervention (30-32,40-42,45).

Thirteen studies involved the use of indirect outcome measures; the data were further extracted using the software of GetData $(18,25,29,31,35-$ $38,40,41,44,47,48)$. The relative data of 6 studies was calculated using the formula described in the above methodology: a). VAS $\%=$ Post-treatment Pain score / Pre-treatment Pain Score $\times 100 \%$; b). VAS reduction rate $(\%)=$ (Post-treatment Pain Score - Pre-treatment Pain Score) / Pre-treatment Pain Score $\times 100 \%$ (26$28,30,32,33)$. The data of the remaining studies were directly extracted from the articles $(34,39,42,43,45)$.

The duration of the rTMS treatment included 10 different sessions: one session (18,25-28,30-33,35-41,4448), 2 sessions $(18,37,44), 3$ sessions $(18,34,37,44), 4$ sessions $(18,35,37,44), 5$ sessions $(18,25,34-37,42-44)$, 6 sessions $(18,37,44), 7$ sessions $(18,37,44), 8$ sessions $(18,37,44), 9$ sessions $(18,37,44)$, and 10 sessions $(18,29,36,37,44)$. The follow-up analgesic effects were observed at one month $(25,34-36,43,44)$, and 2 months $(25,34)$ after the end of 5 repeated rTMS sessions, respectively. The main characteristic of the included studies is listed in Table 2. 
Identified studies from the databases using keywords and bibliographies of relevant articles $(\mathrm{N}=470)$ : PubMed $(\mathrm{N}=160)$, Embase $(\mathrm{N}=293)$, Cochrane Library $(\mathrm{N}=17)$

Exclude duplicate studies $(\mathrm{N}=87)$

Studies remaining after ecxcluding duplicates $(\mathrm{N}=383)$

Exclude upon reading the title and/or absreact $(\mathrm{N}=348)$

Remaining studies $(\mathrm{N}=35)$ evaluated in detail with full text

Additional relevant studies iden-

tified through manual reference $\operatorname{search}(\mathrm{N}=0)$
Excluded studies $(\mathrm{N}=10)$ :

1.useful data can not be contracted

2.no clear description about the parameters of rTMS

Studies included in final analyses $(\mathrm{N}=25)$

Fig. 1. Flow diagram of literature search.

\section{Quality Appraisal}

The results of quality appraisal are shown in Table 1. Apart from the self-controlled studies $(30,32,40$ $42,45)$, randomized allocation of the patients was applied in all other trials. Most of these studies were also double-blind or single-blind. Six studies described the dropout rate $(18,26,27,33,43,44)$. All studies contained the demographic data and were controlled. Two studies $(18,44)$ mentioned the adverse events, such as headache, dizziness, anxiety, etc. However, no study reported serious adverse events related to rTMS treatment.

\section{Meta-analysis}

\section{Primary Outcome}

A total of 32 trials from 25 articles were extracted. This meta-analysis of the pooled analgesic outcome data showed a statistically significant effect size of $-0.86(95 \% \mathrm{Cl},-1.15$ to $-0.56 ; P<0.05)$, suggesting that rTMS was effective in reducing the pain intensity of NP of varying origins as shown in the forest plot of this analysis (Fig. 2). As the associated funnel plot was symmetrical, no publication bias was assumed. However, 
Table 2. Characteristics of the included studies.

\begin{tabular}{|c|c|c|c|c|c|c|c|c|}
\hline & Study & $\begin{array}{c}\text { Mean } \\
\text { Age } \\
\text { (Year) }\end{array}$ & $\begin{array}{c}\text { Mean } \\
\text { Continue } \\
\text { Time } \\
\text { of NP } \\
\text { (Month) } \\
\end{array}$ & $N(E / C)$ & Study Design & $\begin{array}{c}\text { Coil/ } \\
\text { Stimulation } \\
\text { Site }\end{array}$ & Parameters and Dosage & $\begin{array}{l}\text { Outcome } \\
\text { measure }\end{array}$ \\
\hline 1 & $\begin{array}{l}\text { Ahmed } \\
2011\end{array}$ & 52.7 & 33.4 & $27(17 / 10)$ & $\begin{array}{l}\text { Parallel sham } \\
\text { control }\end{array}$ & Figure8/M1 & $\begin{array}{l}20 \mathrm{~Hz}, 80 \% \text { RMT, } 200 \text { pulses } \times 10 \\
\text { train/ session, five sessions days, } \\
\text { ITI }=50 \text { s }\end{array}$ & VAS \\
\hline 2 & $\begin{array}{l}\text { André- } \\
\text { Obadia } \\
2006\end{array}$ & 53 & 82.8 & $12(12 / 12)$ & $\begin{array}{l}\text { Cross-over sham } \\
\text { control }\end{array}$ & Figure8/M1\& & $\begin{array}{l}20 \mathrm{~Hz}, 90 \% \mathrm{RMT}, 80 \text { pulses } \times 20 \\
\text { trains, ITI }=84 \mathrm{~s}\end{array}$ & VAS \\
\hline 3 & $\begin{array}{l}\text { André- } \\
\text { Obadia } \\
2008\end{array}$ & 54.2 & 60 & $28(28 / 28)$ & $\begin{array}{l}\text { Cross-over sham } \\
\text { control }\end{array}$ & Figure8/M1\& & $\begin{array}{l}20 \mathrm{~Hz}, 90 \% \text { RMT, } 80 \text { pulses } \times 20 \\
\text { trains, ITI }=84 \mathrm{~s}\end{array}$ & NRS \\
\hline 4 & $\begin{array}{l}\text { André- } \\
\text { Obadia } \\
2011\end{array}$ & 55 & $>6$ & $45(45 / 45)$ & $\begin{array}{l}\text { Cross-over sham } \\
\text { control }\end{array}$ & Figure8/M1\& & $\begin{array}{l}20 \mathrm{~Hz}, 90 \% \mathrm{RMT}, 80 \text { pulses } \times 20 \\
\text { trains, ITI }=84 \mathrm{~s}\end{array}$ & NRS \\
\hline 5 & Defrin 2007 & 52.0 & 12 & $11(6 / 5)$ & $\begin{array}{l}\text { Parallel sham } \\
\text { control }\end{array}$ & Figure8/M1\& & $\begin{array}{l}5 \mathrm{~Hz}, 115 \% \text { RMT, } 500 \text { pulses } \times \\
1 \text { train/session, } 10 \text { sessions days }\end{array}$ & VAS \\
\hline 6 & Goto 2008 & 63.1 & 61.2 & $17(17 / 17)$ & Self control & Figure8/M1\& & $\begin{array}{l}5 \mathrm{~Hz}, 90 \% \mathrm{RMT}, 50 \text { pulses } \times 10 \\
\text { trains, ITI }=50 \mathrm{~s}\end{array}$ & NRS \\
\hline 7 & $\begin{array}{l}\text { Hirayama } \\
2006\end{array}$ & 56.8 & 76.8 & $20(20 / 20)$ & $\begin{array}{c}\text { Cross-over sham } \\
\text { control }\end{array}$ & Figure8/M1\& & $\begin{array}{l}5 \mathrm{~Hz}, 90 \% \text { RMT, } 50 \text { pulses } \times 10 \\
\text { trains, ITI }=50 \mathrm{~s}\end{array}$ & VAS \\
\hline 8 & $\begin{array}{l}\text { Hosomi } \\
2013^{2} \mathrm{a}\end{array}$ & 61.2 & 56.4 & $29(29 / 29)$ & $\begin{array}{l}\text { Cross-over sham } \\
\text { control }\end{array}$ & Figure 8/M1 & $\begin{array}{l}\text { 5Hz, } 90 \% \text { RMT, } 50 \text { pulses } \times 10 \\
\text { trains/session, } 10 \text { sessions days. } \\
\text { ITI }=50 \text { s, }\end{array}$ & VAS \\
\hline & $\begin{array}{l}\text { Hosomi } \\
2013^{2} b\end{array}$ & 60.1 & 59.5 & $35(35 / 35)$ & $\begin{array}{l}\text { Cross-over sham } \\
\text { control }\end{array}$ & Figure 8/M1 & $\begin{array}{l}5 \mathrm{~Hz}, 90 \% \text { RMT, } 50 \text { pulses } \times 10 \\
\text { trains/session, } 10 \text { sessions days, } \\
\text { ITI }=50 \text { s, }\end{array}$ & VAS \\
\hline 9 & $\begin{array}{l}\text { Hosomi } \\
2013^{1}\end{array}$ & 59.6 & 48.1 & $21(21 / 21)$ & Self control & Figure 8/M1 & $\begin{array}{l}5 \mathrm{~Hz}, 90 \% \mathrm{RMT}, 50 \text { pulses } \times 10 \\
\text { trains/session, ITI }=50 \mathrm{~s}\end{array}$ & VAS \\
\hline \multirow[t]{2}{*}{10} & Jetté 2013a & 50 & 93.6 & $16(16 / 16)$ & $\begin{array}{l}\text { Cross-over sham } \\
\text { control(arm) }\end{array}$ & Figure8/M1* & $\begin{array}{l}10 \mathrm{~Hz}, 90 \% \text { RMT, } 50 \text { pulses } \times 40 \\
\text { trains } / \text { session, ITI }=25 \mathrm{~s}\end{array}$ & NRS \\
\hline & Jetté 2013b & 50 & 93.6 & $16(16 / 16)$ & $\begin{array}{l}\text { Cross-over sham } \\
\text { control(leg) }\end{array}$ & Figure8/M1* & $\begin{array}{l}10 \mathrm{~Hz}, 110 \% \text { RMT, } 50 \text { pulses } \times 40 \\
\text { trains/session, ITI }=25 \mathrm{~s}\end{array}$ & NRS \\
\hline 11 & Kang 2009 & 54.8 & 60.5 & $11(11 / 11)$ & $\begin{array}{l}\text { Cross-over sham } \\
\text { control }\end{array}$ & Figure8/M1\# & $\begin{array}{l}10 \mathrm{~Hz}, 80 \% \text { RMT, } 50 \text { pulses } \times 20 \\
\text { trains/session, } 5 \text { sessions days, } \\
\text { ITI }=55 \mathrm{~s}\end{array}$ & NRS \\
\hline \multirow[t]{2}{*}{12} & $\begin{array}{l}\text { Khedr } \\
2005 \mathrm{a}\end{array}$ & 51.5 & 36 & $24(14,10)$ & $\begin{array}{l}\text { Parallel sham } \\
\text { control(TGN) }\end{array}$ & Figure 8/M1 & $\begin{array}{l}20 \mathrm{~Hz}, 80 \% \text { RMT, } 200 \text { pulses } \times 1 \\
\text { train/session, } 5 \text { sessions days }\end{array}$ & VAS \\
\hline & $\begin{array}{l}\text { Khedr } \\
2005 b\end{array}$ & 52.3 & 18 & $24(14,10)$ & $\begin{array}{l}\text { Parallel sham } \\
\text { control(PSP) }\end{array}$ & Figure 8/M1 & $\begin{array}{l}20 \mathrm{~Hz}, 80 \% \text { RMT, } 200 \text { pulses } \times 1 \\
\text { train/session, } 5 \text { sessions days }\end{array}$ & VAS \\
\hline 13 & Khedr 2014 & 47.5 & 16.1 & $30(15 / 15)$ & $\begin{array}{l}\text { Parallel sham } \\
\text { control }\end{array}$ & Figure 8/M1 & $\begin{array}{l}20 \mathrm{~Hz}, 80 \% \text { RMT, } 200 \text { pulses } \times 10 \\
\text { trains/session, ten session days, } \\
\text { ITI }=30 \mathrm{~s}\end{array}$ & VAS \\
\hline 14 & $\begin{array}{l}\text { Lefaucheur } \\
2001^{1}\end{array}$ & 57.2 & $\ldots$ & $14(14 / 14)$ & $\begin{array}{l}\text { Cross-over sham } \\
\text { control }\end{array}$ & Figure 8/M1 & $\begin{array}{l}10 \mathrm{~Hz}, 80 \% \text { RMT, } 50 \text { pulses } \times 20 \\
\text { trains/session, } 12 \text { sessions days, } \\
\text { ITI }=55 \mathrm{~s}\end{array}$ & VAS \\
\hline 15 & $\begin{array}{l}\text { Lefaucheur } \\
2001^{2}\end{array}$ & 54.7 & $\ldots$ & $18(18 / 18)$ & $\begin{array}{l}\text { Cross-over sham } \\
\text { control }\end{array}$ & Figure 8/M1 & $\begin{array}{l}10 \mathrm{~Hz}, 80 \% \text { RMT, } 50 \text { pulses } \times 20 \\
\text { trains } / \text { session, ITI }=55 \mathrm{~s}\end{array}$ & VAS \\
\hline
\end{tabular}


High Frequency Transcranial Magnetic Stimulation Therapy For Neuropathic Pain

Table 2 (cont.). Characteristics of the included studies.

\begin{tabular}{|c|c|c|c|c|c|c|c|c|}
\hline & Study & $\begin{array}{l}\text { Mean } \\
\text { Age } \\
\text { (Year) }\end{array}$ & $\begin{array}{c}\text { Mean } \\
\text { Continue } \\
\text { Time } \\
\text { of NP } \\
\text { (Month) } \\
\end{array}$ & $\mathbf{N}(\mathbf{E} / \mathrm{C})$ & Study Design & $\begin{array}{c}\text { Coil/ } \\
\text { Stimulation } \\
\text { Site }\end{array}$ & Parameters and Dosage & $\begin{array}{l}\text { Outcome } \\
\text { measure }\end{array}$ \\
\hline 16 & $\begin{array}{l}\text { Lefaucheur } \\
2006\end{array}$ & 55.5 & 64.8 & $44(22 / 22)$ & $\begin{array}{c}\text { Parallel sham } \\
\text { control }\end{array}$ & Figure 8/M1 & $\begin{array}{l}10 \mathrm{~Hz}, 90 \% \mathrm{RMT}, 60 \text { pulses } \times 20 \\
\text { trains/session, ITI }=54 \mathrm{~s}\end{array}$ & VAS \\
\hline 17 & $\begin{array}{l}\text { Lefaucheur } \\
2008\end{array}$ & 54.2 & $>12$ & $46(46 / 46)$ & $\begin{array}{l}\text { Cross-over sham } \\
\text { control }\end{array}$ & Figure 8/M1 & $\begin{array}{l}10 \mathrm{~Hz}, 90 \% \mathrm{RMT}, 60 \text { pulses } \times 20 \\
\text { trains/session, ITI }=54 \mathrm{~s}\end{array}$ & VAS \\
\hline \multirow[t]{3}{*}{18} & $\begin{array}{l}\text { Lefaucheur } \\
\text { 2012a }\end{array}$ & 53.8 & 49.2 & $14(14 / 14)$ & Self control-rTMS & Figure8/M1* & $\begin{array}{l}10 \mathrm{~Hz}, 90 \% \text { AMT, } 100 \text { pulses } \times 20 \\
\text { trains/session, ITI }=30 \mathrm{~s}\end{array}$ & VAS \\
\hline & $\begin{array}{l}\text { Lefaucheur } \\
2012 b\end{array}$ & 53.8 & 49.2 & $14(14 / 14)$ & $\begin{array}{c}\text { Self } \\
\text { control-iTBS-rTMS }\end{array}$ & Figure8/M1* & $\begin{array}{l}10 \mathrm{~Hz}, 90 \% \text { AMT, } 100 \text { pulses } \times \\
20 \text { trains/session, ITI }=30 \mathrm{~s}\end{array}$ & VAS \\
\hline & $\begin{array}{l}\text { Lefaucheur } \\
2012 c\end{array}$ & 53.8 & 49.2 & $14(14 / 14)$ & $\begin{array}{c}\text { Self } \\
\text { control-cTBS-rTMS } \\
\end{array}$ & Figure8/M1* & $\begin{array}{l}10 \mathrm{~Hz}, 90 \% \text { AMT, } 100 \text { pulses } \times \\
20 \text { trains/session, ITI }=30 \mathrm{~s}\end{array}$ & VAS \\
\hline 19 & $\begin{array}{l}\text { Matsumura } \\
2013\end{array}$ & 63.6 & $>3$ & $20(20 / 20)$ & $\begin{array}{l}\text { Cross-over sham } \\
\text { control }\end{array}$ & Figure 8/M1 & $\begin{array}{l}5 \mathrm{~Hz}, 100 \% \text { RMT, } 50 \text { pulses } \times 10 \\
\text { trains } / \text { session, ITI }=25 \mathrm{~s}\end{array}$ & VAS \\
\hline 20 & Ohn 2012 & 50.9 & 21.9 & $14(14 / 14)$ & Self control & Figure 8/M1 & $\begin{array}{l}10 \mathrm{~Hz}, 90 \% \text { RMT, } 50 \text { pulses } \times 20 \\
\text { trains/session, } 5 \text { sessions days, } \\
\text { ITI }=55 \mathrm{~s}\end{array}$ & VAS \\
\hline \multirow[t]{2}{*}{21} & $\begin{array}{l}\text { Onesti } \\
2013 a\end{array}$ & 70.6 & $\ldots$ & $11(11 / 11)$ & $\begin{array}{l}\text { Cross-over sham } \\
\text { control }\end{array}$ & Figure H/M1 & $\begin{array}{l}20 \mathrm{~Hz}, 100 \% \text { RMT, } 50 \text { pulses } \times 30 \\
\text { trains/session, } 5 \text { sessions days, } \\
\text { ITI }=30 \mathrm{~s}\end{array}$ & VAS \\
\hline & $\begin{array}{l}\text { Onesti } \\
2013 b\end{array}$ & 70.6 & $\ldots$ & $12(12 / 12)$ & $\begin{array}{l}\text { Cross-over sham } \\
\text { control }\end{array}$ & Figure H/M1 & $\begin{array}{l}20 \mathrm{~Hz}, 100 \% \text { RMT, } 50 \text { pulses } \times 30 \\
\text { trains/session, } 5 \text { sessions days, } \\
\text { ITI }=30 \mathrm{~s}\end{array}$ & VAS \\
\hline 22 & $\begin{array}{l}\text { Picarelli } \\
2010\end{array}$ & 42.1 & 80.1 & $22(11,11)$ & $\begin{array}{l}\text { Parallel sham } \\
\text { control }\end{array}$ & Figure 8/M1 & $\begin{array}{l}10 \mathrm{~Hz}, 100 \% \mathrm{RMT}, 100 \text { pulses } \\
\times 25 \text { trains/session, } 10 \text { sessions } \\
\text { days, ITI }=58 \mathrm{~s} \text {. }\end{array}$ & VAS \\
\hline 23 & Pleger 2004 & 51.0 & 35.0 & $10(10 / 10)$ & Self control & Figure8/M1* & $\begin{array}{l}10 \mathrm{~Hz}, 110 \% \mathrm{RMT}, 12 \text { pulses } \times 10 \\
\text { trains/session, ITI }=10 \mathrm{~s} .\end{array}$ & VAS \\
\hline 24 & $\begin{array}{l}\text { Rollink } \\
2002\end{array}$ & 51.3 & 32.4 & $12(12 / 12)$ & $\begin{array}{l}\text { Cross-over sham } \\
\text { sequence-control }\end{array}$ & Figure8/M1* & $\begin{array}{l}20 \mathrm{~Hz}, 80 \% \text { RMT, } 40 \text { pulses } \times 20 \\
\text { trains } / \text { session, ITI }=58 \mathrm{~s}\end{array}$ & VAS \\
\hline \multirow[t]{2}{*}{25} & $\begin{array}{l}\text { Saitoh } \\
\text { 2007a }\end{array}$ & 59.4 & 122.4 & $13(13 / 13)$ & $\begin{array}{c}\text { Cross-over sham } \\
\text { control }\end{array}$ & Figure 8/M1 & $\begin{array}{l}5 \mathrm{~Hz}, 90 \% \mathrm{RMT}, 50 \text { pulses } \times 10 \\
\text { trains/session, ITI }=50 \mathrm{~s}\end{array}$ & VAS \\
\hline & $\begin{array}{l}\text { Saitoh } \\
2007 b\end{array}$ & 59.4 & 122.4 & $13(13 / 13)$ & $\begin{array}{l}\text { Cross-over sham } \\
\text { control }\end{array}$ & Figure 8/M1 & $\begin{array}{l}10 \mathrm{~Hz}, 90 \% \mathrm{RMT}, 100 \text { pulses } \times 5 \\
\text { trains/session, ITI }=50 \mathrm{~s}\end{array}$ & VAS \\
\hline
\end{tabular}

M1: Primary motor cortex corresponding to a painful region; M1*: Primary motor cortex contralateral to the painful site or to the dominant-hand site; M1\#: the right primary motor cortex; M1\&: no mention the specific site of coil; Figure 8: figure-of- 8 coil; Figure H: figure-of- $\mathrm{H}$ coil; N: no. of patients; E: experimental group; C: control group; RMT: the resting motor threshold; AMT: the active motor threshold; ITI: inter-train interval; VAS: visual analgesic scale; NRS: numerical rating scales; VAS (\%) = Post-treatment Pain Score / Pre-treatment Pain Score; VAS reduction rate (\%) $=($ Post-treatment Pain Score - Pre-treatment Pain Score $) /$ Pre-treatment Pain Score; .... no available data could be extracted from that article.

because of the substantial heterogeneity $(12=81 \%)$, further subgroup analysis, stratified by rTMS stimulation frequency, the number of treatment sessions, and the follow-up observation were conducted.

Stratified analysis by rTMS stimulation frequency $(5 \mathrm{~Hz}, 10 \mathrm{~Hz}$, and $20 \mathrm{~Hz})$ did not reduce the heterogeneity, suggesting similar efficacy of pain reduction induced by these HF-rTMS treatments (Fig. 3).
Fig. 4 shows that one session of rTMS treatment was significantly effective in reducing the pain intensity of NP (the pooled SMD $=-0.94[95 \% \mathrm{Cl},-1.28$ to $-0.61 ; P$ $<0.001]$ ). After excluding the single rTMS data in Fig. 4 , the effective size of one session of rTMS treatment was $0.54,95 \% \mathrm{Cl}-1.01$ to $-0.08, P<0.01$. The relative symmetrical funnel plot suggested little publication bias. Increasing the treatment session from 2 to 10 also 
Pain Physician: November/December 2015; 18:E1029-E1046

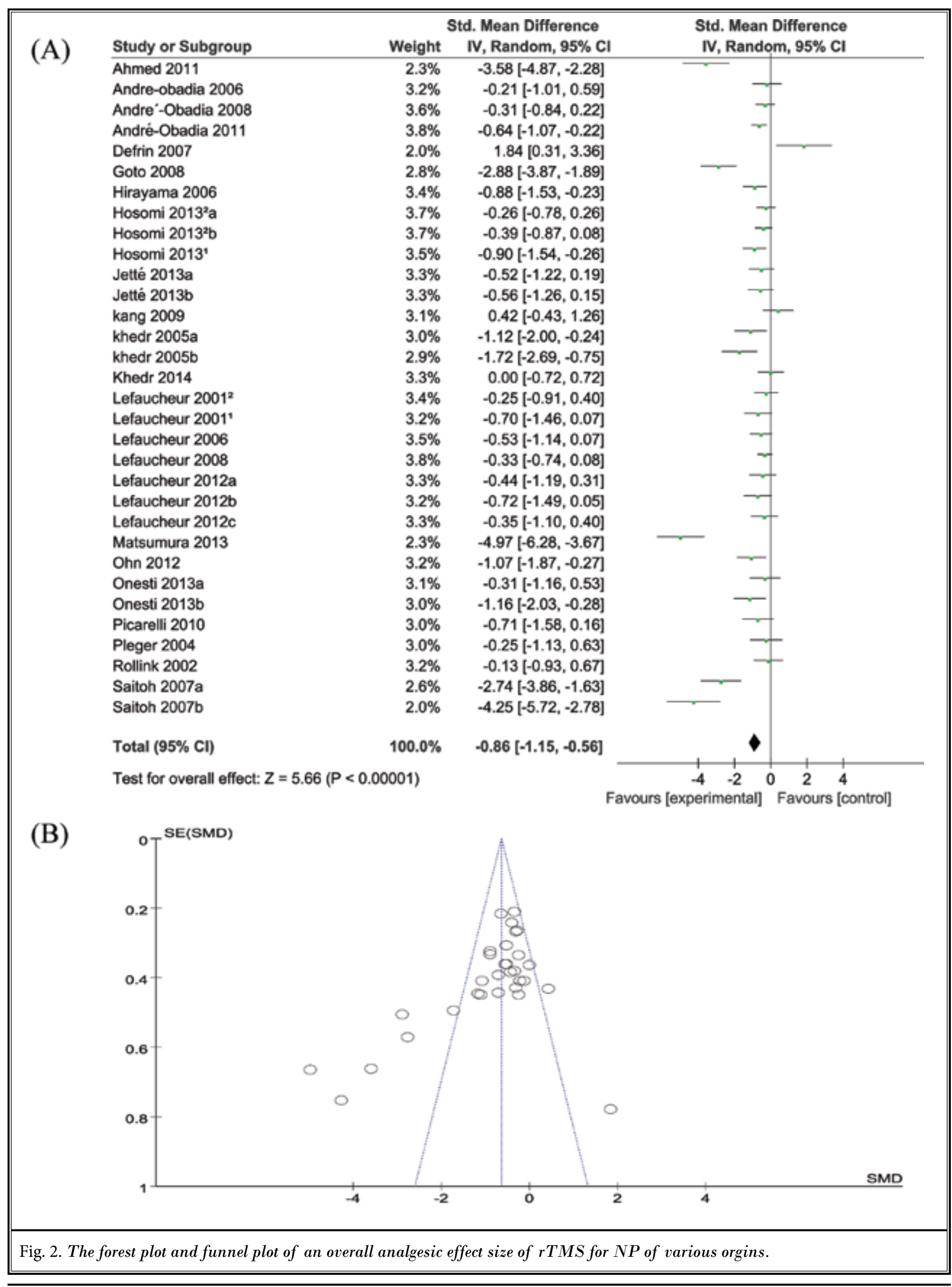


High Frequency Transcranial Magnetic Stimulation Therapy For Neuropathic Pain

Study or Subgroup

1.1.1 $5 \mathrm{~Hz}$

Defrin 2007

Goto 2008

Hirayama 2006

Hosomi $2013^{2} a$

Hosomi $2013^{2} b$

Hosomi 2013'

Matsumura 2013

Saitoh 2007a

Subtotal $(95 \% \mathrm{Cl})$

Test for overall effect: $Z=2.88(P=0.004)$

1.1.2 $10 \mathrm{~Hz}$

Jetté 2013a

Jetté 2013b

kang 2009

Lefaucheur 2001²

Lefaucheur 2001"

Lefaucheur 2006

Lefaucheur 2008

Lefaucheur $2012 \mathrm{a}$

Lefaucheur 2012b

Lefaucheur 2012c

Ohn 2012

Picarelli 2010

Pleger 2004

Saitoh 2007 b

Subtotal $(95 \% \mathrm{Cl})$

Test for overall effect: $Z=3.64(P=0.0003)$

\subsubsection{Hz}

Ahmed 2011

Andre-obadia 2006

Andre'-Obadia 2008

André-Obadia 2011

khedr 2005a

khedr 2005b

Khedr 2014

Onesti 2013a

Onesti 2013b

Rollink 2002

Subtotal $(95 \% \mathrm{Cl})$

Test for overall effect: $Z=3.34(P=0.0008)$

Total $(95 \% \mathrm{Cl})$

Test for overall effect: $Z=5.66$ ( $P<0.00001)$

Test for subgroup differences: $\mathrm{Chi}^{2}=2.62, \mathrm{df}=2(\mathrm{P}=0.27), \mathrm{I}^{2}=23.7 \%$
Std. Mean Difference

Weight IV, Random, $95 \% \mathrm{Cl}$

$2.0 \%$

$2.8 \%$

$3.4 \%$

$3.7 \%$

$3.7 \%$

$3.5 \%$

$2.3 \%$

$2.6 \%$

$23.9 \%$

$3.3 \%$

$3.3 \%$

$3.1 \%$

$3.4 \%$

$3.2 \%$

$3.5 \%$

$3.8 \%$

$3.3 \%$

$3.2 \%$

$3.3 \%$

$3.2 \%$

$3.0 \%$

$3.0 \%$

$2.0 \%$

$44.7 \%$

$-0.52[-1.22,0.19]$

$-0.56[-1.26,0.15]$

$0.42[-0.43,1.26]$

$-0.25[-0.91,0.40]$

$-0.70[-1.46,0.07]$

$-0.53[-1.14,0.07]$

$-0.33[-0.74,0.08]$

$-0.44[-1.19,0.31]$

$-0.72[-1.49,0.05]$

$-0.35[-1.10,0.40]$

$-1.07[-1.87,-0.27]$

$-0.71[-1.58,0.16]$

$-0.25[-1.13,0.63]$

$-4.25[-5.72,-2.78]$

$-0.59[-0.91,-0.27]$

$-3.58[-4.87,-2.28]$

$-0.21[-1.01,0.59]$

$-0.31[-0.84,0.22]$

$-0.64[-1.07,-0.22]$

$-1.12[-2.00,-0.24]$

$-1.72[-2.69,-0.75]$

$0.00[-0.72,0.72]$

$-0.31[-1.16,0.53]$

$-1.16[-2.03,-0.28]$

$-0.13[-0.93,0.67]$

$-0.80[-1.26,-0.33]$

$31.4 \%$

$100.0 \%$
Std. Mean Difference

IV, Random, $95 \% \mathrm{Cl}$

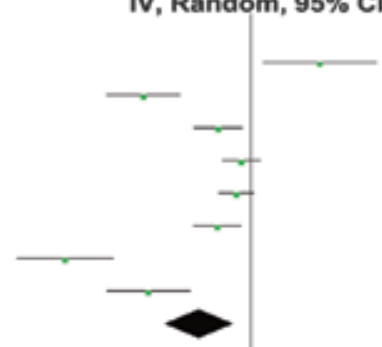

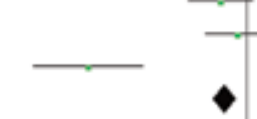

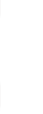

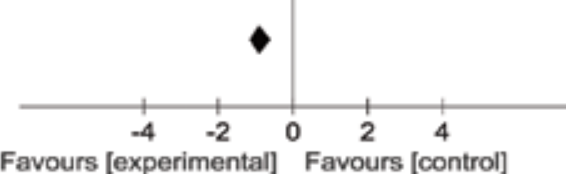

Favours [experimental] Favours [control]

Fig. 3. The subgroup analyses of rTMS frequency: all 3 high frequency $r T M S(5,10$, and $20 \mathrm{~Hz})$ produced similar analgesic effects in NP patients. 
Pain Physician: November/December 2015; 18:E1029-E1046

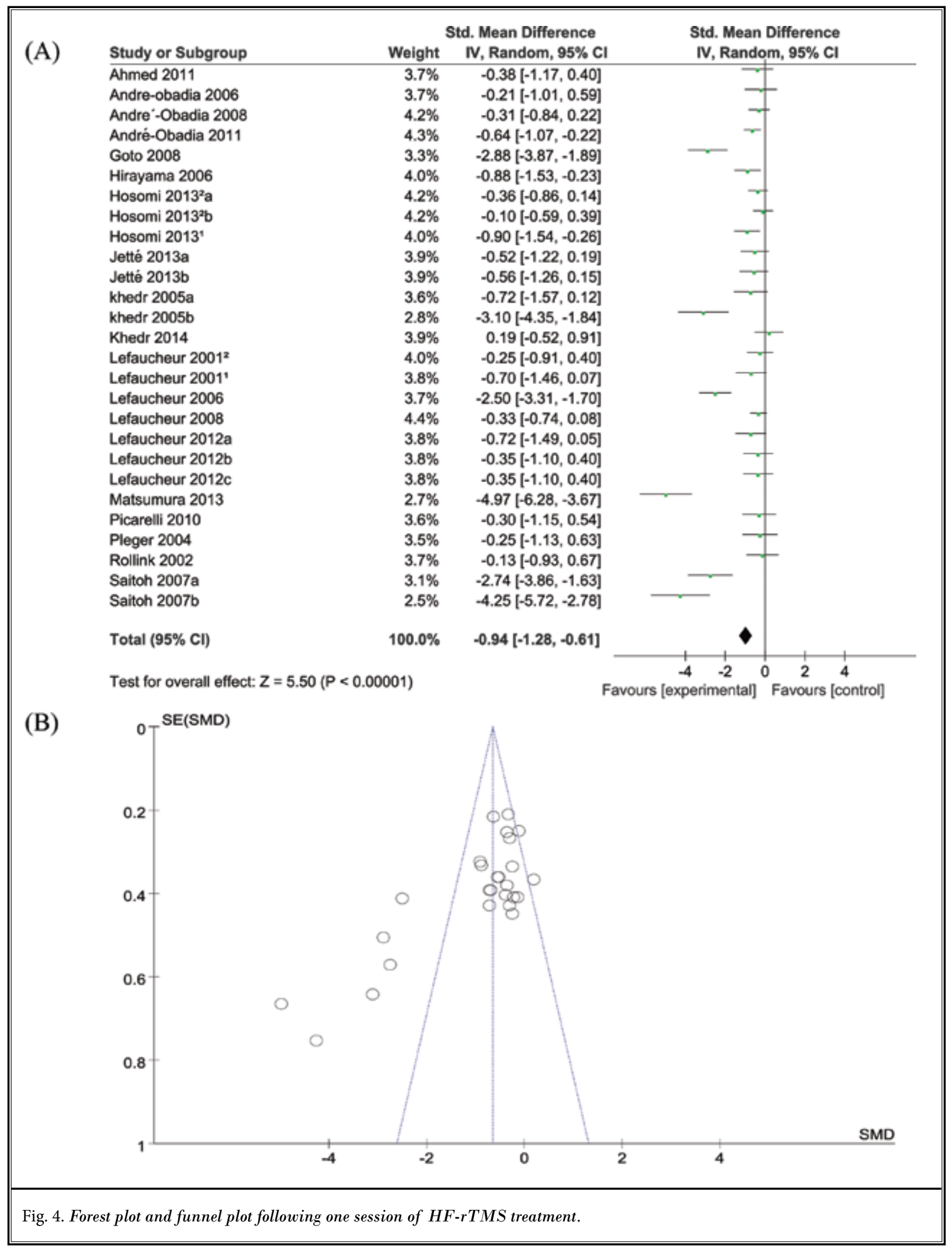


produced significant pain reduction (Figs. 5A-C, 6A-B, 7A-D), with the average maximal pain reduction found after 5 sessions of rTMS treatment (SMD $=-1.22,95 \% \mathrm{Cl}$, -1.87 to $-0.57 ; P<0.001$ ) (Fig. $6 \mathrm{~A}$ ).

\section{Secondary Outcome}

To better evaluate the long-term analgesic effect of rTMS, the data were extracted from studies containing 5 sessions of treatment. Five studies $(25,34,35,43,44)$ with follow-up data at one month after rTMS, 2 studies with 2 months data $(25,34)$, and one study with 3 months data (44) were included for analysis. The pooled analgesic effect was significant at one month after rTMS (SMD $=-0.96,95 \% \mathrm{Cl},-1.55$ to $-0.37, P<0.05)$,

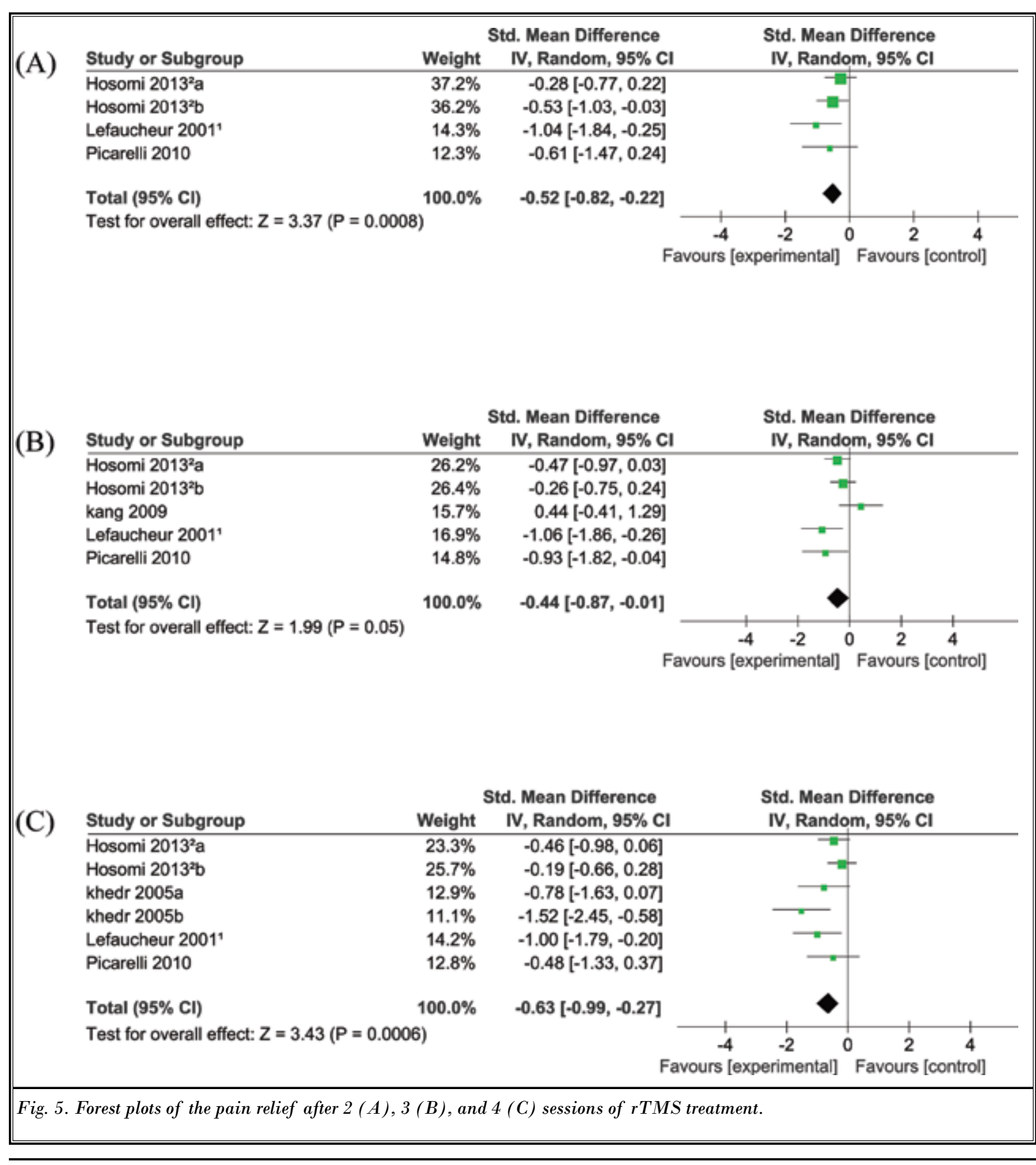




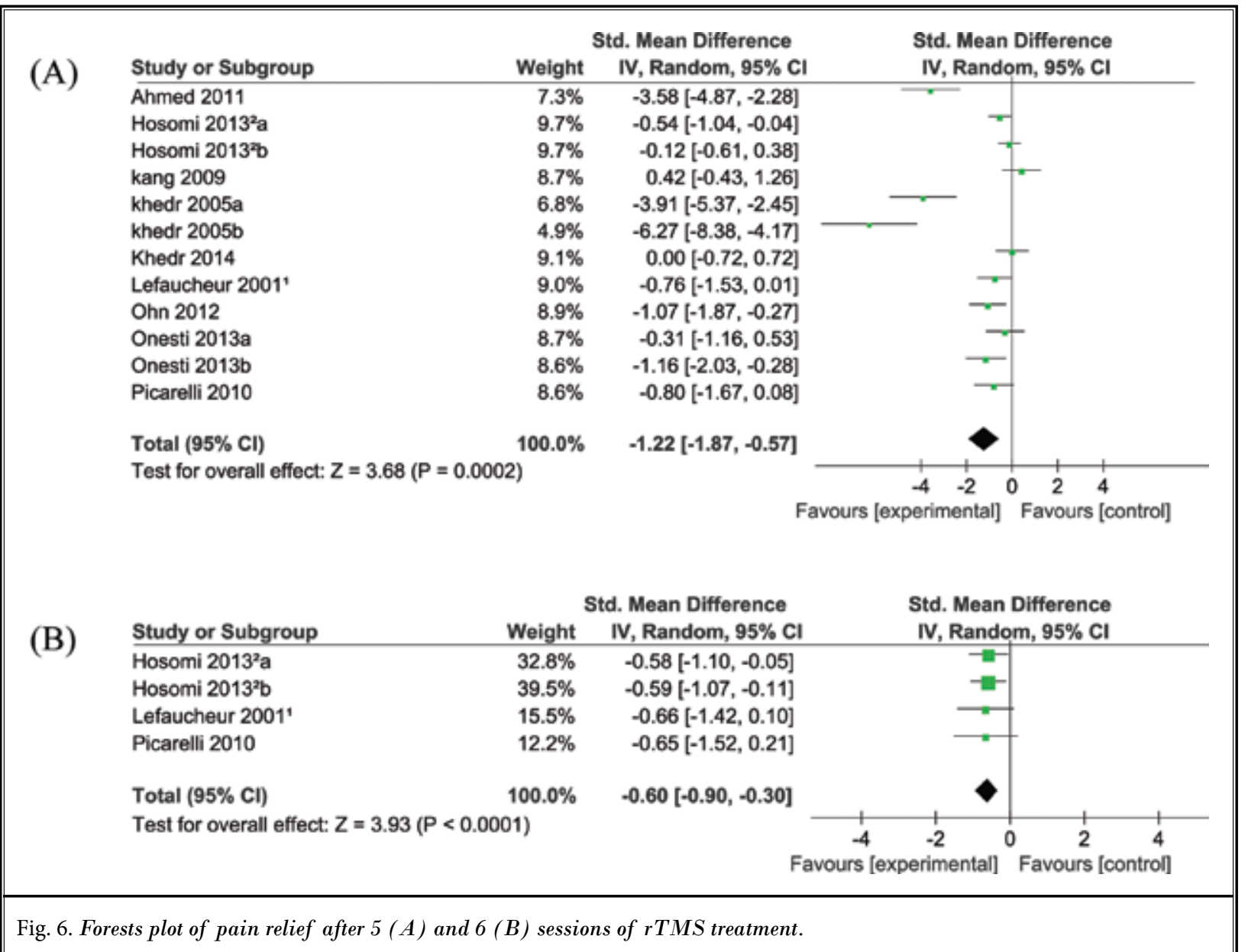

but not so at 2 months after rTMS (SMD = -1.05 (95\% Cl, -2.15 to $0.05, P>0.05$ ) (Fig. 8). It should be noted that the data of 2 and 3 months follow-up was too limited to be computed for a valid conclusion.

\section{Discussion}

To the best of the current knowledge, this is the first meta-analysis to assess the optimal parameters of rTMS therapy for NP. Our analyses show that although one session of rTMS treatment is effective in pain reduction, greater pain relief occurred after 5 sessions of rTMS treatment. Furthermore, the pain relieving effect of multiple rTMS (5 sessions) treatment can be maintained for at least one month.

So far there is not enough clinical evidence to determine the long-term effect of rTMS therapy (Ionger than 2 months post-treatment). In Picarelli et al's trial (44), no persistent difference in pain reduction was found after 3 months follow-up between rTMS therapy and sham controls. It is conceivable that if the acute effects of rTMS parameters can be established for NP, it will pave the way for more labor intensive and more costly long observation studies of rTMS therapy trials in future

This results are consistent with the views recently expressed by Lefaucheur et al (49) and Ohn et al (42), who proposed that rTMS could be a useful therapeutic method for NP when multiple sessions of rTMS intervention were given. A recent NP study has shown that 5 daily sessions of $20 \mathrm{~Hz}$ rTMS over the motor cortex produced long-lasting pain relief in patients with phantom pain (25). In a comprehensive review, Leo et al also suggested that multiple sessions of high frequency rTMS on motor cortex could relieve pain (50). Thus, current findings suggest that multiple sessions of rTMS are effective for relieving chronic pain of NP. 
High Frequency Transcranial Magnetic Stimulation Therapy For Neuropathic Pain

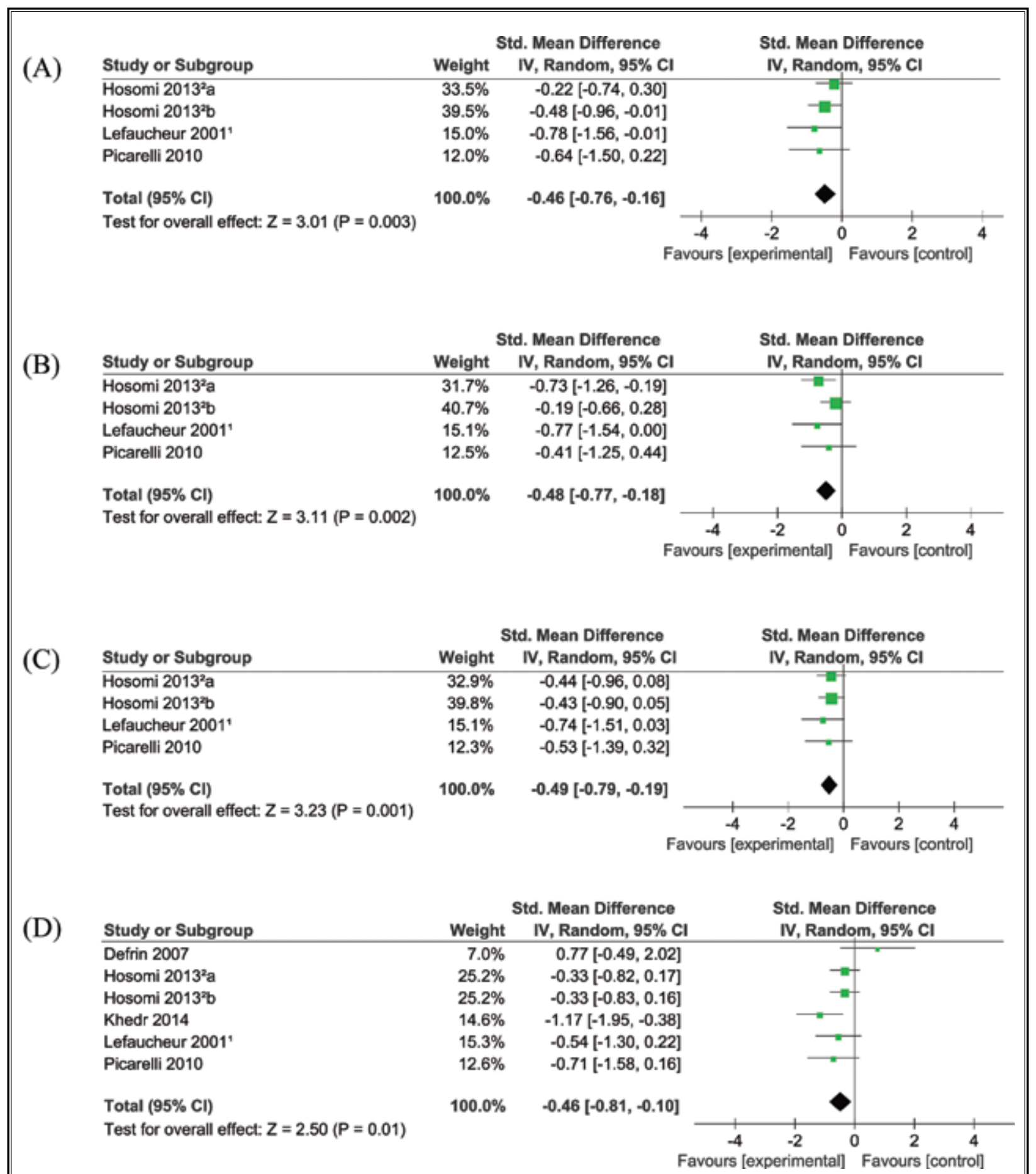

Fig. 7. Forest plot after 7 (A), 8 (B), 9 (C), and 10 (D) sessions of rTMS treatment. 


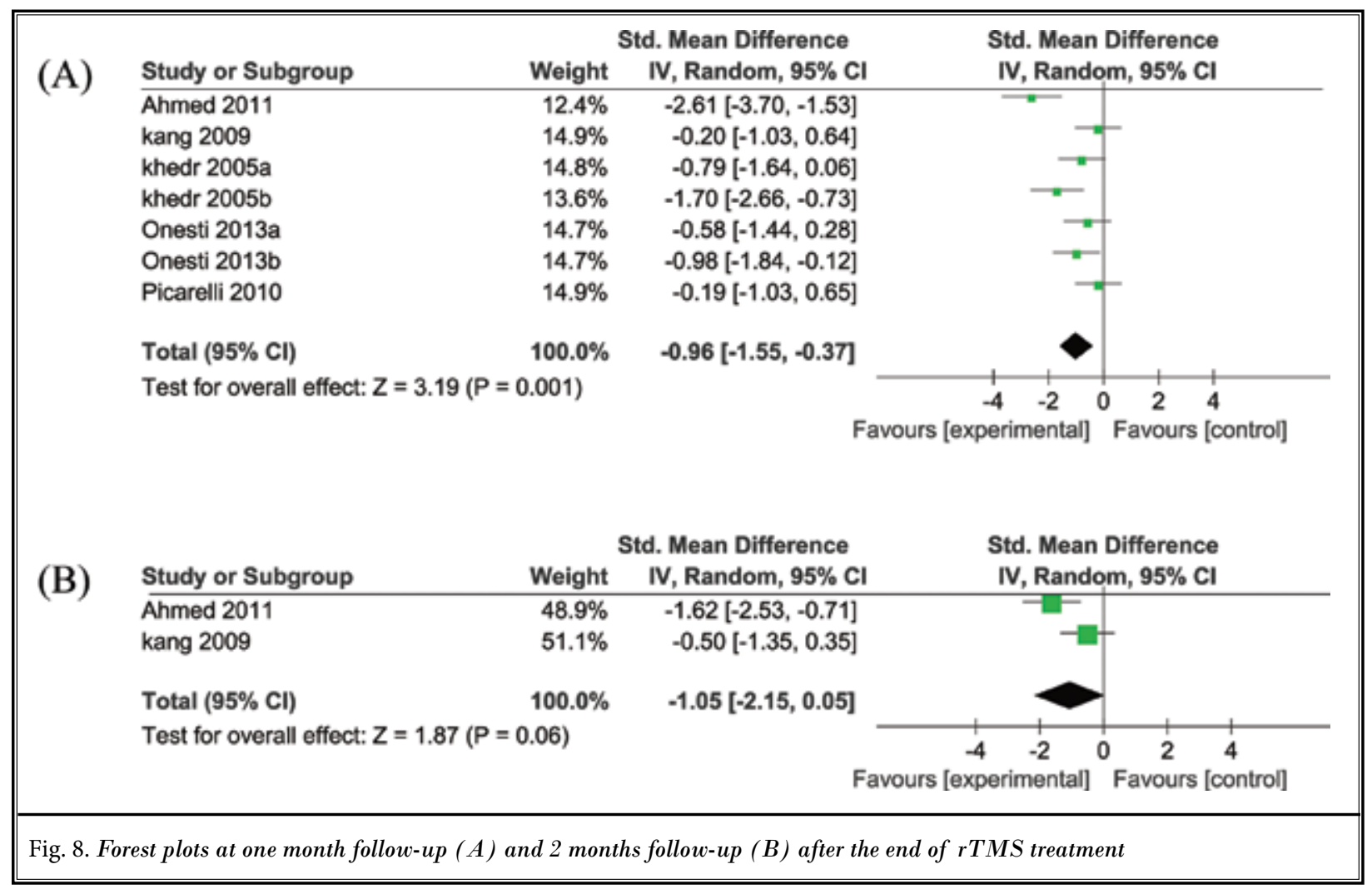

In this meta-analysis, only the HF-rTMS trials were included, and no different pain-alleviating effects were presented among $5 \mathrm{~Hz}, 10 \mathrm{~Hz}$, and $20 \mathrm{~Hz} \mathrm{HF}-\mathrm{rTMS}$. It is known that HF-rTMS enhances neuronal firing efficacy whereas low-frequency rTMS (LF-rTMS) has the opposite effect (51). Lefaucheur et al (48) studied the pain-relief effect of rTMS at 0.5 and $10 \mathrm{~Hz}$ in 18 patients with intractable unilateral hand pain. They concluded that $10 \mathrm{~Hz}$ rTMS, but not $0.5 \mathrm{~Hz}$ rTMS was effective in pain reduction. In a separate study Lefaucheur et al (39) showed that HF-rTMS could improve thermal sensory perception in the painful region of NP and the improved warm sensation perception was correlated with pain relief only after HF-rTMS but not after low frequency. Saitoh et al (47) tested rTMS therapy at $1 \mathrm{~Hz}, 5 \mathrm{~Hz}$, and $10 \mathrm{~Hz}$ in $13 \mathrm{NP}$ patients, and showed that $10 \mathrm{~Hz}$ rTMS was more effective in pain relief than $5 \mathrm{~Hz}$ rTMS, while $1 \mathrm{~Hz}$ rTMS was ineffective. Similar findings of effective pain control by high frequency rTMS $(5-20 \mathrm{~Hz})$ have also been reported by multiple labs $(18,43,52)$. However, Sampson et al (53) reported that 4 of 9 patients had significant pain relief, and 3 of them were rapid onset of relief, after $1 \mathrm{~Hz}$ low frequency rTMS for 5 days per week for 3 weeks.
Thus prolonged repeated LF-rTMS could still be a potentially optional analgesic therapy for NP patients who are insensitive to HF-rTMS.

There are 2 main central ascending pain pathways: the laterospinothalamic tract and the medial lemniscal pathway (paleospinothalamic). The laterospinothalamic tract projects to the insular and to the somatosensory cortex; the other to the insular, anterior cingulated cortex, and prefrontal cortex. The ipsilateral and/or contralateral M1 of the pain site was selected in most rTMS studies. Hirayama et al (31) concluded that the M1 was the best target for rTMS treatment of intractable pain, in spite of the fact that M1, the postcentral gyrus (S1), premotor area (preM), and supplementary motor area (SMA) are located adjacently. Sampsonet al (53) and Borckardt et al (54) showed that stimulation of the prefrontal cortex also had an analgesic effect.

The mechanism underlying the analgesic effect of rTMS is still unknown. Studies of motor cortex stimulation (MCS) suggested that MCS may result in direct inhibition of brain regions involved in the emotional response to pain and/or induced indirect mechanisms that will trigger descending inhibitory pathway to act at the dorsal horn level. rTMS may have a similar 


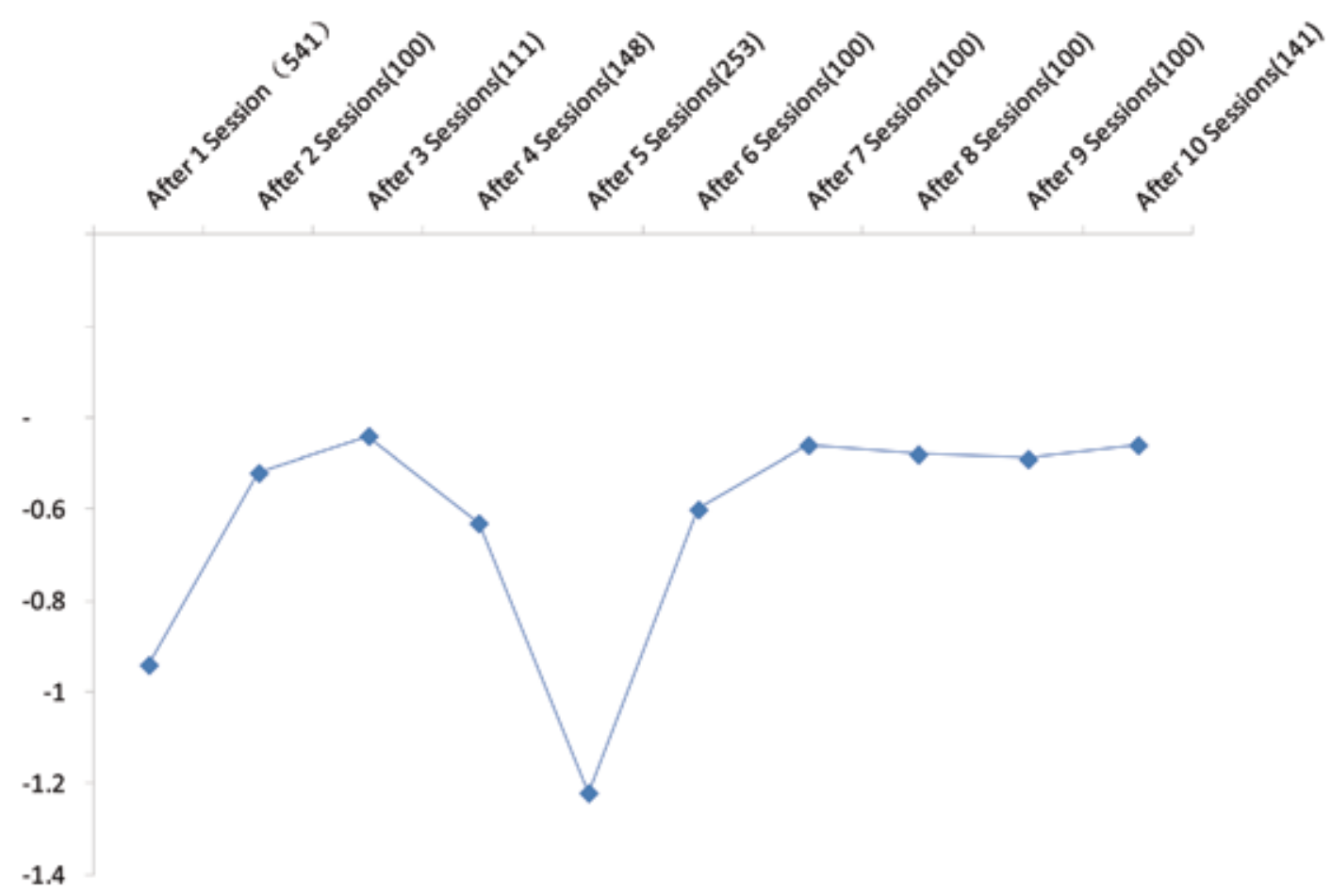

Fig. 9. The effect sizes (Y-axis) over the course of the different points of treatment ( $X$-axis). The digits in parentheses represent numbers of patients corresponding for each forest plot.

mechanism to that of MCS (16). Another possibility was that rTMS relieved the pain through improving the blood flow of the affected area. It is known that there is a relative decrease in cerebral blood flow ( $\mathrm{rCBF}$ ) during chronic pain (55), and rTMS stimulation over the M1 significantly increased $\mathrm{rCBF}$ in NP patients in positron emission tomography. (PET) studies $(55,56)$.

Several functional magnetic resonance imaging (fMRI) studies of post-stroke central pain showed significantly decreased activity in the secondary somatosensory cortex, including the insula, prefrontal cortex, and putamen in rTMS responders, whereas no change was noted in nonresponders (42). A functional imaging study by Goto et al (30), tracking the fibers of the corticospinal tract (CST) and thalamocortical tract (TCT) using diffusion tensor imaging (DTI), suggested the importance of the intactness of CST and TCT for rTMS-induced pain reduction as the rTMS-effective responders had higher delineation ratio of the CST and the TCT than the rTMS-ineffective responders. Likewise, the results of Leung et al's (16) meta-analysis also pointed to the importance of the overall intact- ness of the pain modulatory pathways in affecting the potential analgesic effect of rTMS. In Ohn et al's report (42), the integrity of the superior TCT in the ipsilesional hemisphere showed significant correlation with changes of VAS score after rTMS. In addition, Ahmed et al (25) inferred that VAS score reduction induced by rTMS of the M1 was correlated with an increase in cerebral beta-endorphin, which is known to be an analgesic factor in the nervous system. In short, the plastic changes from the structure and function of brain areas relative to emotion induced by rTMS may be connected to pain relief. In spite of these experimental data, the therapeutic mechanism of rTMS for NP remains speculative at this stage.

Although analysis of different rTMS sessions (Fig. 9) revealed greater analgesic effect of one and 5 sessions of rTMS treatment, respectively, it is noted that 6 out of 27 one-session trials showed exceptional analgesic response after rTMS. After excluding these 6 single session rTMS data, the effective size of one session rTMS treatment was reduced to $0.42,95 \% \mathrm{Cl}$ -0.56 to -0.28 , but still at a significant level $(P<0.01)$. 
Similarly, although the 5-session rTMS treatment induced the maximal analgesic effect, 3 out of the 12 5 -session trials showed exceptional analgesic response to rTMS treatment. And excluding these 3 trials has reduced the analgesic effect of 5-session rTMS to a level similar to that of other sessions of rTMS. Furthermore, we found that these trials with exceptional analgesic response to 5-session HF-rTMS were conducted in patients with central post-stroke pain $(25,35,42)$. This suggests that the differential analgesic effect of rTMS may depend on the neuroanatomical origins of the NP pathophysiology, with more effective rTMS treatment response in NP originated from the "top" (supraspinal, cranial, or spinal) rather than the "bottom" (nerve root or peripheral nerve) (16). Overall, HF-rTMS appears to be a promising effective and alternative treatment for certain chronic NP, especially if its analgesic effects can be proven in NP patients who are resistant to drug treatment.

\section{Conclusion}

Although single session HF-rTMS is effective in acute pain relief of NP, multiple sessions of HF-rTMS ( 5 sessions) could be more effective for relieving the pain of NP, especially for post-stroke NP. Because only a limited number of trials evaluated the long-term effects of HF-rTMS at one and 2 months post rTMS treatment, further large-scale controlled trials including clearly defined pain subgroups with both acute and long-term observations (up to 3 months) are needed to validate and expand the current findings and to reveal the underlying mechanisms of rTMS.

\section{Acknowledgments}

This work was supported by the National Natural Science Foundation of China (NSFC, No.81271559); the State Administration of Foreign Experts Affairs, the P.R. of China (No. SZD201313); the Basic Program Funded by Science \& Technology Department of Sichuan Province (No.12JC0565); and the Scientific Research Project of Sichuan Health Department (No.110616). The sponsors had no role in study design, data collection and analysis, writing of the manuscript, or decision to submit the article for publication.

\section{References}

1. Attal N, Bennet D, Treede RD. Neuropathic pain update: From basic mechanisms to clinical management. Tracey I, editor. Pain 2012 - refresher courses 14th World Congress on Pain; IASP, Seattle 2012, pp 85-110.

2. Treede RD, Jensen TS, Campbell JN, Cruccu G, Dostrovsky JO, Griffin JW, Hansson $P$, Hughes R, Nurmikko T, Serra J. Neuropathic pain: Redefinition and a grading system for clinical and research purposes. Neurology 2008; 70:1630-1635.

3. Dieleman JP, Kerklaan J, Huygen FJ, Bouma PA, Sturkenboom MC. Incidence rates and treatment of neuropathic pain conditions in the general population. Pain 2008; 137:681-688.

4. Torrance N, Smith BH, Bennett MI, Lee AJ. The epidemiology of chronic pain of predominantly neuropathic origin. Results from a general population survey.] Pain 2006; 7:281-289.

5. Bouhassira $D$, Lanteri-Minet $M$, Attal $\mathrm{N}$, Laurent B, Touboul C. Prevalence of chronic pain with neuropathic characteristics in the general population. Pain
2008; 136:380-387.

6. Szczudlik A, Dobrogowski J, Wordliczek J, Stepien A, Krajnik M, Leppert W, Woron J, Przeklasa-Muszynska A, Kocot-Kepska M, Zajaczkowska R, Janecki M, Adamczyk A, Malec-Milewska M. Diagnosis and management of neuropathic pain: Review of literature and recommendations of the Polish Association for the study of pain and the Polish Neurological Society - Part one. Neurol Neurochir Pol 2014; 48:262-271.

7. Finnerup NB, Otto $M$, McQuay HJ, Jensen TS, Sindrup SH. Algorithm for neuropathic pain treatment: An evidence based proposal. Pain 2005; 118:289-305.

8. Hansson PT, Attal N, Baron R, Cruccu G. Toward a definition of pharmacoresistant neuropathic pain. Eur J Pain 2009; 13:439-440.

9. Navarro X, Vivo M, Valero-Cabre A. Neural plasticity after peripheral nerve injury and regeneration. Prog Neurobiol 2007; 82:163-201.

10. Basbaum Al, Bautista DM, Scherrer G, Julius D. Cellular and molecular mechanisms of pain. Cell 2009; 139:267-284.
11. Saitoh Y, Yoshimine T. Stimulation of primary motor cortex for intractable deafferentation pain. Acta Neurochir Suppl 2007; 97:51-56.

12. Rosa MA, Lisanby SH. Somatic treatments for mood disorders. Neuropsychopharmacol 2012; 37:102-116.

13. Rossi S, Hallett M, Rossini PM, PascualLeone A. Safety, ethical considerations, and application guidelines for the use of transcranial magnetic stimulation in clinical practice and research. Clin Neurophysiol 2009; 120:2008-2039.

14. Siebner HR, Rothwell J. Transcranial magnetic stimulation: New insights into representational cortical plasticity. Exp Brain Res 2003; 148:1-16.

15. Lefaucheur JP. The use of repetitive transcranial magnetic stimulation (rTMS) in chronic neuropathic pain. Neurophysiol Clin 2006; 36:117-124.

16. Leung A, Donohue M, Xu R, Lee R, Lefaucheur JP, Khedr EM, Saitoh Y, AndreObadia N, Rollnik J, Wallace M, Chen R. rTMS for suppressing neuropathic pain: A meta-analysis. J Pain 2009; 10:1205-1216. 
17. O'Connell NE, Wand BM, Marston L, Spencer S, DeSouza LH. Non-invasive brain stimulation techniques for chronic pain. Cochrane Collaboration 2014; 4:CDoo8208

18. Hosomi K, Shimokawa T, Ikoma K, Nakamura Y, Sugiyama K, Ugawa Y, Uozumi T, Yamamoto T, Saitoh Y. Daily repetitive transcranial magnetic stimulation of primary motor cortex for neuropathic pain: A randomized, multicenter, double-blind, crossover, sham-controlled trial. Pain 2013; 154:1065-1072.

19. Merskey H, Bogouk N. Detailed descriptions of pain syndromes. In: Classification of Chronic Pain. IASP, Seattle, 1994, pp 59-71

20. Siddall PJ. Management of neuropathic pain following spinal cord injury: Now and in the future. Spinal Cord 2009; 47:352-359.

21. Price DD, McGrath PA, Rafii A, Buckingham $B$. The validation of visual analogue scales as ratio scale measures for chronic and experimental pain. Pain 1983; 17:45-56.

22. Farrar JT, Young JP, LaMoreaux L, Werth $J L$, Poole RM. Clinical importance of changes in chronic pain intensity measured on an 11-point numerical pain rating scale. Pain 2001; 94:149-158.

23. Moher D, Schulz KF, Altman D. The CONSORT statement: Revised recommendations for improving the quality of reports of parallel-group randomized trials. JAMA 2001; 285:1987-1991.

24. Borenstein M, Hedges LV, Higgins JP.T, Rothstein HR. Fixed-Effect Versus Ramdon-Effects Models. In: Borenstein M, Hedges LV, Higgins JP.T, Rothstein HR. Introduction to Meta-Analysis. 1st ed. John Wiley and Sons, Ltd, Chichester, 2009, pp77-79

25. Ahmed MA, Mohamed SA, Sayed D. Long-term antalgic effects of repetitive transcranial magnetic stimulation of motor cortex and serum beta-endorphin in patients with phantom pain. Neurol Res 2011; 33:953-958.

26. André-Obadia N, Peyron R, Mertens $P$, Mauguière $F$, Laurent B, Garcia-Larrea L. Transcranial magnetic stimulation for pain control. Double-blind study of different frequencies against placebo, and correlation with motor cortex stimulation efficacy. Clin Neurophysiol 2006; 117:1536-1544

27. Andre-Obadia N, Mertens P, Gueguen A, Peyron R, Garcia-Larrea L. Pain relief by rTMS: Differential effect of current flow but no specific action on pain subtypes. Neurology 2008; 71:833-840.

28. André-Obadia N, Magnin M, GarciaLarrea L. On the importance of placebo timing in rTMS studies for pain relief. Pain 2011; 152:1233-1237.

29. Defrin R, Grunhaus L, Zamir D, Zeilig $G$. The effect of a series of repetitive transcranial magnetic stimulations of the motor cortex on central pain after spinal cord injury. Arch Phys Med Rehabil 2007; 88:1574-1580.

30. Goto $\mathrm{T}$, Saitoh $\mathrm{Y}$, Hashimoto $\mathrm{N}, \mathrm{Hi}-$ rata $\mathrm{M}$, Kishima $\mathrm{H}$, Oshino $\mathrm{S}$, Tani $\mathrm{N}$, Hosomi K, Kakigi R, Yoshimine T. Diffusion tensor fiber tracking in patients with central post-stroke pain; correlation with efficacy of repetitive transcranial magnetic stimulation. Pain 2008; 140:509-518.

31. Hirayama A, Saitoh $Y$, Kishima H, Shimokawa T, Oshino S, Hirata M, Kato A, Yoshimine T. Reduction of intractable deafferentation pain by navigationguided repetitive transcranial magnetic stimulation of the primary motor cortex. Pain 2006; 122:22-27.

32. Hosomi K, Kishima H, Oshino S, Hirata M, Tani N, Maruo T, Yorifuji S, Yoshimine T, Saitoh Y. Cortical excitability changes after high-frequency repetitive transcranial magnetic stimulation for central poststroke pain. Pain 2013; 154:1352-1357.

33. Jette F, Cote I, Meziane HB, Mercier C. Effect of single-session repetitive transcranial magnetic stimulation applied over the hand versus leg motor area on pain after spinal cord injury. Neurorehabil Neural Repair 2013; 27:636-643.

34. Kang BS, Shin HI, Bang MS. Effect of repetitive transcranial magnetic stimulation over the hand motor cortical area on central pain after spinal cord injury. Arch Phys Med Rehabil 2009; 90:1766-1771.

35. Khedr EM, Kotb $H$, Kamel NF, Ahmed MA, Sadek R, Rothwell JC. Long-lasting analgic effects of daily sessions of repetitive transcranial magnetic stimulation in central and peripheral neuropathic pain. J Neurol Neurosur Ps 2005; 76:833-838.

36. Khedr EM, Kotb HI, Mostafa MG, Mohamad MF, Amr SA, Ahmed MA, Karim AA, Kamal SM. Repetitive transcranial magnetic stimulation in neuropathic pain secondary to malignancy: A randomized clinical trial. Eur J Pain 2014; 19:519-527.
37. Lefaucheur J, Drouot X, Nguyen J. Interventional neurophysiology for pain control: Duration of pain relief following repetitive transcranial magnetic stimulation of the motor cortex. Neurophysiol Clin 2001; 31:247-252.

38. Lefaucheur J, Drouot X, Menard-Lefaucheur I, Keravel Y, Nguyen J. Motor cortex rTMS restores defective intracortical inhibition in chronic neuropathic pain. Neurology 2006; 67:1568-1574.

39. Lefaucheur J, Drouot X, Menard-Lefaucheur I, Keravel Y, Nguyen J. Motor cortex rTMS in chronic neuropathic pain: Pain relief is associated with thermal sensory perception improvement. J Neurol Neurosur Ps 2008; 79:1044-1049.

40. Lefaucheur JP, Ayache S, Sorel M, Farhat $\mathrm{W}$, Zouari $\mathrm{H}$, Ciampi de Andrade D, Ahdab R, Ménard-Lefaucheur I, Brugieres $P$, Goujon C. Analgesic effects of repetitive transcranial magnetic stimulation of the motor cortex in neuropathic pain: Influence of theta burst stimulation priming. Eur J Pain 2012; 16:1403-1413.

41. Matsumura $\mathrm{Y}$, Hirayama T, Yamamoto T. Comparison between pharmacologic evaluation and repetitive transcranial magnetic stimulation-induced analgesia in poststroke pain patients. Neuromodulation 2013; 16:349-354.

42. Ohn SH, Chang WH, Park C-H, Kim ST, Lee JI, Pascual-Leone A, Kim Y-H. Neural correlates of the antinociceptive effects of repetitive transcranial magnetic stimulation on central pain after stroke. Neurorehab Neural Re 2012; 26:344-352.

43. Onesti E, Gabriele M, Cambieri C, Ceccanti M, Raccah R, Di Stefano G, Biasiotta A, Truini A, Zangen A, Inghilleri $\mathrm{M}$. $\mathrm{H}$-coil repetitive transcranial magnetic stimulation for pain relief in patients with diabetic neuropathy. Eur J Pain 2013; 17:1347-1356.

44. Picarelli $\mathrm{H}$, Teixeira MJ, de Andrade DC, Myczkowski ML, Luvisotto TB, Yeng LT, Fonoff ET, Pridmore S, Marcolin MA. Repetitive transcranial magnetic stimulation is efficacious as an add-on to pharmacological therapy in complex regional pain syndrome (CRPS) type I. J Pain 2010; 11:1203-1210.

45. Pleger B, Janssen F, Schwenkreis P, Völker B, Maier C, Tegenthoff M. Repetitive transcranial magnetic stimulation of the motor cortex attenuates pain perception in complex regional pain syndrome type I. Neurosci Lett 2004; 356:87-90.

46. Rollnik JD, Wüstefeld S, Däuper J, Karst M, Fink M, Kossev A, Dengler R. Repeti- 
tive transcranial magnetic stimulation for the treatment of chronic pain-a pilot study. Eur Neurol 2001; 48:6-10.

47. Saitoh $Y$, Hirayama A, Kishima $H$, Shimokawa T, Oshino S, Hirata M, Tani $\mathrm{N}$, Kato A, Yoshimine T. Reduction of intractable deafferentation pain due to spinal cord or peripheral lesion by highfrequency repetitive transcranial magnetic stimulation of the primary motor cortex. J Neurosurg 2007; 107:555-559.

48. Lefaucheur JP, Drouot X, Keravel $Y$, Nguyen JP. Pain relief induced by repetitive transcranial magnetic stimulation of precentral cortex. Neuroreport 2001; 12:2963-2965.

49. Lefaucheur JP, Antal A, Ahdab R, Ciampi de Andrade D, Fregni F, Khedr EM, Nitsche M, Paulus W. The use of repetitive transcranial magnetic stimulation (rTMS) and transcranial direct current stimulation (tDCS) to relieve pain. Brain
Stimul 2008; 1:337-344.

50. Leo RJ, Latif T. Repetitive transcranial magnetic stimulation (rTMS) in experimentally induced and chronic neuropathic pain: A review. J Pain 2007; 8:453-459.

51. Pell GS, Roth Y, Zangen A. Modulation of cortical excitability induced by repetitive transcranial magnetic stimulation: Influence of timing and geometrical parameters and underlying mechanisms. Prog Neurobiol 2011; 93:59-98.

52. Yilmaz B, Kesikburun S, Yasar E, Tan AK. The effect of repetitive transcranial magnetic stimulation on refractory neuropathic pain in spinal cord injury. J Spinal Cord Med 2014; 37:397-400.

53. Sampson SM, Kung S, McAlpine DE, Sandroni P. The use of slow-frequency prefrontal repetitive transcranial magnetic stimulation in refractory neuro- pathic pain. J ECT 2011; 27:33-37.

54. Borckardt JJ, Smith AR, Reeves ST, Madan A, Shelley N, Branham R, Nahas Z, George MS. A pilot study investigating the effects of fast left prefrontal rTMS on chronic neuropathic pain. Pain Med 2009; 10:840-849.

55. Garcia-Larrea L, Peyron R, Mertens P, Gregoire MC, Lavenne F, Le Bars D, Convers P, Mauguiere F, Sindou $M$, Laurent B. Electrical stimulation of motor cortex for pain control: A combined PET-scan and electrophysiological study. Pain 1999; 83:259-273.

56. Saitoh $Y$, Osaki Y, Nishimura H, Hirano S, Kato A, Hashikawa K, Hatazawa J, Yoshimine $T$. Increased regional cerebral blood flow in the contralateral thalamus after successful motor cortex stimulation in a patient with poststroke pain. J Neurosurg 2004; 100:935-939. 